\title{
Lessons from other diseases: granulomatous inflammation in leishmaniasis
}

\author{
Paul M. Kaye ${ }^{1} \cdot$ Lynette Beattie $^{2}$
}

Received: 12 August 2015 / Accepted: 2 November 2015 / Published online: 17 December 2015

(C) The Author(s) 2015. This article is published with open access at Springerlink.com

\begin{abstract}
The Leishmania granuloma shares some, though not all, properties with that formed following mycobacterial infection. As a simplified, noncaseating granuloma composed of relatively few and largely mononuclear cell populations, it provides a tractable model system to investigate intragranuloma cellular dynamics, immune regulation, and antimicrobial resistance. Here, the occurrence of granulomatous pathology across the spectrum of leishmaniasis, in humans and animal reservoir hosts, is first described. However, this review focuses on the process of hepatic granuloma formation as studied in rodent models of visceral leishmaniasis, starting from the initial infection of Kupffer cells to the involution of the granuloma after pathogen clearance. It describes how the application of intravital imaging and the use of computational modeling have changed some of our thoughts on granuloma function, and illustrates how host-directed therapies have been used to manipulate granuloma form and function for therapeutic benefit. Where appropriate, lessons that may be equally applicable across the spectrum of granulomatous diseases are highlighted.
\end{abstract}

This article is a contribution to the Special Issue on Immunopathology of Mycobacterial Diseases - Guest Editor: Stefan Kaufmann

Electronic supplementary material The online version of this article (doi:10.1007/s00281-015-0548-7) contains supplementary material, which is available to authorized users.

Paul M. Kaye

paul.kaye@york.ac.uk

1 Centre for Immunology and Infection, Department of Biology and Hull York Medical School, University of York, Heslington, York YO10 5DD, UK

2 QIMR Berghofer Medical Research Institute, 300 Herston Rd, Herston, Queensland, Australia 4006
Keywords Leishmania · Granuloma · Mouse models · Intravital imaging $\cdot$ Computational models

\section{Leishmaniasis: a complex granulomatous disease}

The leishmaniases represent some 15 or so human diseases associated with infection by protozoan parasites of the genus Leishmania. Classification of the leishmaniases is not straightforward. Often referred to as a spectrum of diseases, this term implies a continuum in the clinical spectrum associated with a single etiological agent, e.g., as seen for Mycobacterium leprae infection [1]. Most forms of leishmaniasis do show a spectrum of responses after infection, from the maintenance of an asymptomatic subclinical infection through to the expression of overt clinical disease with varying degrees of severity. But for the most part, this spectrum exists independently of the well-described clinical boundaries that distinguish cutaneous, metastatic, and visceral manifestations of the disease. Hence, it may be more informative to refer to the leishmaniases as a "collection" of distinct diseases with different etiological agents, each with characteristic and different immune responses and host-pathogen interactions and each having a spectrum of clinical responses [2, 3]. Different species (and subgenera) of Leishmania characterize disease in the Old World (Europe, Asia, Africa) and New World (the Americas), reflecting a combination of evolutionary adaptation and geographic dispersal of Leishmania parasites and their hosts [4, 5]. Granulomas have been identified following infection with all species of Leishmania, often associated with the curative phase of infection. 


\section{Granulomas as hallmarks of protective immunity: an overview}

Some of the most comprehensive studies of the Leishmania granuloma were conducted by Ridley and Ridley in the early 1980s, who compared different forms and stages of human cutaneous leishmaniasis using a similar approach to that adopted in their pioneering histopathological description of the leprosy spectrum.

They defined many features still relevant today [1]; granulomatous inflammation, mainly showing signs of focal macrophage activation, with or without epitheliod cells, and parasite destruction, in the absence of neutrophil infiltration; and granulomas with evidence of macrophage/monocyte lysis and the presence, albeit transiently, of neutrophils leading to focal necrosis. However, a more contemporary study of CL cases found a surprisingly high number of caseating granulomas (equating to the Ridley group C) [6] It is important to note that the CL lesion has all the hallmarks of a "wound" and as such, the histopathological features of wound healing, including necrotic granulation tissue, fibrinogenesis, and sloughing off of the lesion, occur in parallel with the antimicrobial functions that are associated with granulomatous inflammation. In other words, "lesion" healing may be independent from parasite killing. This notion is supported by the observation that in genetic crosses of Leishmania major-susceptible and Leishmania major-resistant BALB/c and C57BL/6 mice, genetic loci associated with disease severity did not associate with levels of local immune response, including the extent of granulomatous inflammation $[7,8]$.

Granulomas with evident multinucleated and epitheliod cells are associated with good in vitro recall responses (measured using the lymphocyte transformation test, LTT) and a requirement for less drug to achieve healing in Leishmania braziliensis infection, whereas the presence of necrosis and a polymorph infiltrate was contraindicated [9]. Lymphocyte, plasma cells, and occasional eosinophils also predominate in granulomas in simian models of CL. In L. braziliensis infections in the Rhesus monkey (Macaca mulatta), chronic granulomas with all the normal human cellular constituents (Langhans giant cells, epitheliod cells, plasma cells, parasitecontaining macrophages, lymphocytes) are seen, and at the final stages of healing there is fibroblast infiltration and fibrotic substitution $[10,11]$.

In contrast to the skin, the characteristic wound healing response is rather minimal in systemic sites, providing the opportunity to examine the antimicrobial functions of granulomas largely independently from this process. However, detailed studies of granulomas in human systemic disease are few and far between, reflecting the difficulties in sampling these sites for histopathological investigation. In humans, the histopathology of active clinical kala-azar most commonly reflects what might be seen as a failure of granulomatous inflammation, being associated with extensive lymphoid tissue remodeling, splenomegaly/lymphadenopathy, and elevated levels of plasma cells [12]. Nevertheless, in some cases, fibrin-ring granulomas (so named for the donut like appearance due to a ring of fibrinoid material) may be evident in the liver [13]. A retrospective analysis of liver biopsies in Iran also suggests that hepatic granulomas may form in some patients with visceral leishmaniasis [14]. Mirroring the absence of granulomas in the spleen of active visceral leishmaniasis (VL) cases, epitheliod granulomas, common in the lymph nodes of CL patients, are also not usually present in Sudanese VL patients who present with lymphadenopathy [15]. Granulomas, usually poorly formed, are also sparse in the bone marrow [16] [17]. In contrast, asymptomatic patients have been reported to have epitheliod granulomas in the liver [18].

In Rhesus monkeys, however, the kinetics of hepatic granuloma formation has been amenable to study. Six weeks postexperimental infection with $2 \times 10^{7}$ amastigotes $/ \mathrm{kg}$ of body weight of a virulent Leishmania infantum strain, poorly differentiated granulomas were observed, largely comprising aggregates of macrophages and lymphocytes. In contrast, by 24 weeks, granuloma organization with the occurrence of Langhans-type multinucleated cells was observed, with evident presence of amastigotes. Although this time course of infection was not extended to determine the rate of involution of granulomas under these infection conditions, animals receiving an experimental vaccine for VL (comprising Ad5expresing A2 antigen boosted with rA2+IL-12+alum) showed granuloma resolution at week 24 along with reduced parasite burden. In the spleens of infected animals, a similar pattern to human disease was noted, including heavily infected macrophages in the subcapsular region and red pulp, disorganized follicular structures, and sinusoidal congestion. Again, vaccinated animals had more normal follicular histology.

In canine VL, both skin [19] and hepatic [20] granuloma formation has been extensively characterized, demonstrating a similar trend to that observed in humans and macaques. Thus, asymptomatic dogs with low parasite burdens had histopathological evidence of robust granuloma formation in the liver, with evidence of recruitment of effector $\mathrm{T}$ cells and local macrophage/dendritic cell activation. Granulomas were either not evident or not organized in the liver of symptomatic dogs with high parasite burdens [20]. Of note, whereas tissue response correlated well with parasite load and immune status in the liver, this appeared less evident in the spleen, mirroring the organ-specific dichotomy of responses observed in murine models of VL (see below).

In hamsters, intradermal promastigote inoculation lead to neutrophil influx over the first $48 \mathrm{~h}$ followed by a mononuclear cell-rich granuloma that was coincident with clearance of parasites from the skin $[21,22]$. Nevertheless, this local 
response did not stop dissemination and the ultimate development of VL in this model. It was also suggested from these studies that visceral dissemination might need to occur before granuloma formation [21].

Mice infected intravenously with amastigotes of Leishmania donovani or L. infantum have provided multiple insights into the fine detail of granuloma formation [23], as described in the following sections. Nevertheless, it is worth noting here that mice represent something of a hybrid when it comes to modeling the response observed in humans. Whereas the granulomatous response in the hepatic microenvironment appears to follow many of the characteristics observed in subclinical human and canine infection and in the ultimately self-resolving infection in primates, the spleen fails to make a robust granulomatous response and shows hallmarks of progressive human, primate, and canine disease. This expression of organ-specific immunity, within the same genetically and microbiota-defined host, provides a clear example of the compartmentalization of immune responses based on tissue-specific environmental factors [12, 24].

Post kala-azar dermal leishmaniasis is an important but relatively understudied sequelae of visceral leishmaniasis [25-28]. In Sudan, where post-kala-azar dermal leishmaniasis (PKDL) is commonly self-limiting, granulomas were reported in $\sim 20 \%$ of patients, though other signs of chronic inflammation, e.g., the presence of giant cells, were more frequently observed [29, 30]. In India, where PKDL is less frequent but more chronic in nature, granuloma formation was notably absent, suggestive of a causative relationship between granuloma formation and host protection [31].

\section{Initiation of the hepatic granulomatous response in mice}

Experimental murine models of VL have played an important role in understanding the development of granulomatous inflammation in the hepatic microenvironment. It should be emphasized at the outset, however, that this represents very much a model system and that many aspects of the biology of natural infection are omitted from the model. Natural sandfly-mediated transmission of small numbers of Leishmania metacyclic promastigotes into the dermis is replaced by the intravenous administration of large (often very large!) numbers of culture-derived promastigotes (most common when using Leishmania chagasi/infantum) or amastigotes (commonly used for $L$. donovani). Although new imaging approaches have highlighted the novel route of Plasmodium transmission to the lymphatics during mosquito blood feeding [32], our current knowledge of sandfly transmission of Leishmania suggests that parasites are deposited in the dermis, where they may be engulfed by neutrophils, dermal macrophages, or dermal DCs [3]. Sandfly salivary components also have a direct capacity to modulate local and perhaps systemic immune responses, and this may alter the hepatic microenvironment. Whether visceralisation of Leishmania to the liver and elsewhere is accomplished by free parasites (unlikely) or by migratory infected myeloid cells (more likely) has yet to be determined experimentally. In part, this technical challenge is one of numbers - tracing a few initiating foci of infected Kupffer cells within the myriad sinusoidal network of the liver is a major challenge.

Hence, what we know about the initiation of granulomatous inflammation in the liver begins from the somewhat artificial starting point of an interaction between free amastigotes and liver resident cells lining the sinusoidal spaces of the healthy non-inflamed liver. Under these infection conditions, the Kupffer cell is the primary target of $L$. donovani amastigotes. Early immunohistochemical analysis identified Kupffer cells on the basis of their characteristic morphology and anatomical position within the sinusoids [33]. Later, using real-time intravital 2-photon imaging, the uptake of amastigotes in situ was visualized for the first time. Kupffer cells are the resident tissue macrophages of the liver, originating from a wave of primitive yolk sac hematopoiesis that starts around embryonic day E7.5 and involves a $m y b$-independent precursor [34, 35]. Using a LysMcre reporter mouse crossed to the dual-color reporter mouse line $\mathrm{mT} / \mathrm{mG}$, Beattie et al. demonstrated that Kupffer cells are stationary within the sinusoids and display elegant membrane activity, with continuously extending and retracting lamellipodia activity [36, 37]. Following contact with injected amastigotes, uptake is rapid and completed within minutes, with no obvious change in lamellipodia activity either at the contact site or distal to it (Fig. 1a; Supplementary Movie 1 and [36]. Phagocytosed amastigotes were observed to reside within intracellular vacuoles from the earliest timepoint that can be studied (Fig. 1b and Supplementary Movie 2; Beattie, unpublished).

Uptake of Leishmania by macrophages has been shown to be dependent on both host cell type and parasite species and involve distinct mechanisms in vitro [38, 39]. The intravital imaging data described above demonstrate that within the liver microenvironment, phagocytosis of amastigotes by Kupffer cells is a relatively passive process, displaying none of the indicators of induced phagocytosis or macropinocytosis. A currently unanswered, yet tractable question that follows from these imaging studies is the nature of the ligand responsible for amastigotes recognition and uptake. In vitro studies have identified a range of potential receptor ligand pairs involved in Leishmania-macrophage interactions and their relevance in vivo deserves to be explored more fully [38]. A recent study identified divergent roles for the C-type lectins SIGNR3, dectin1, and mannose receptor in determining the fate of L. infantum. Whereas SIGNR3 favored parasite survival through selective modulation of LTB4-IL-1 $\beta$, both dectin1 and MR were coupled to signaling pathways associated with 

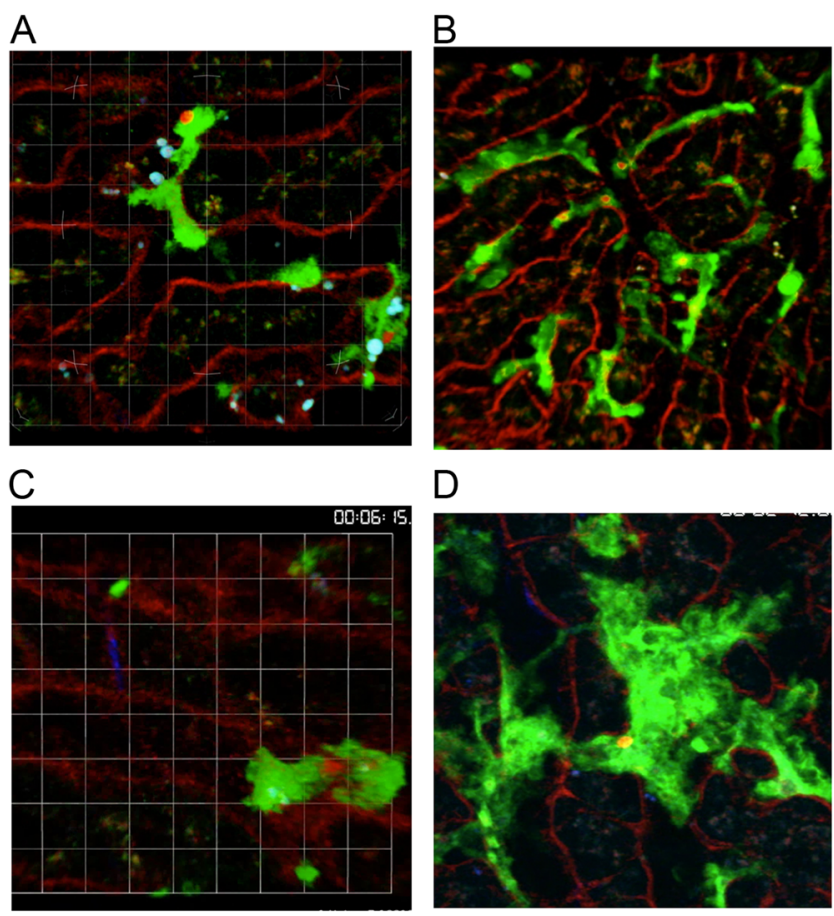

D

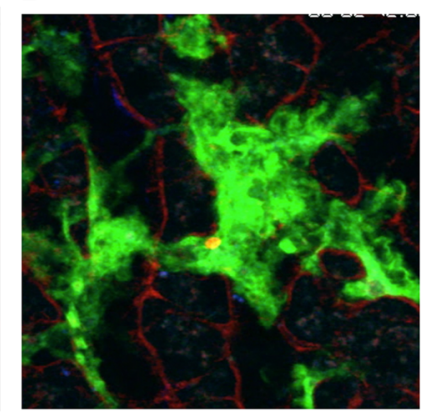

$\mathrm{E}$

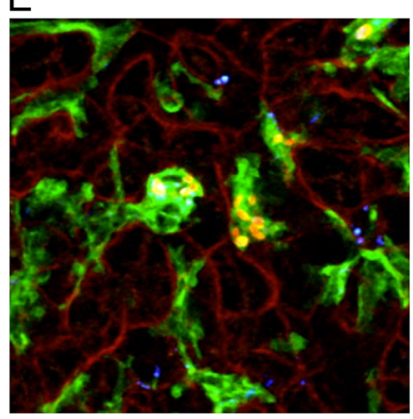

$\mathrm{F}$
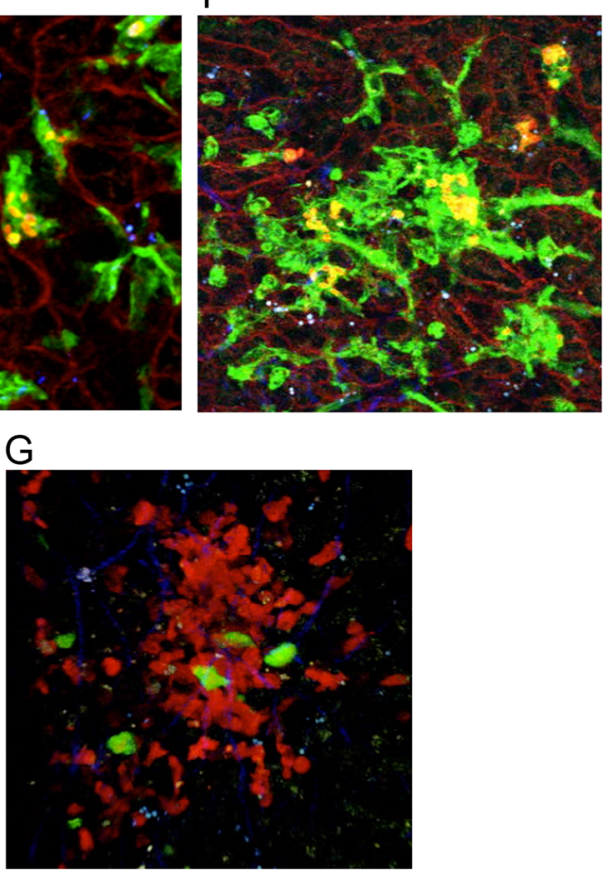

Fig. 1 Intravital imaging of granuloma formation in experimental L. donovani infection. a-g Intravital analysis of $\mathrm{KC}$ (green) in liver sinusoids (red) in $(\mathrm{mT} / \mathrm{mG} \times \text { LysMcre })_{\mathrm{F} 1}$ mice; a immediately following intravenous injection of tdTomato $L$. donovani amastigotes, $\mathbf{b} 2 \mathrm{~h}$ postinjection of tdTomato $L$. donovani amastigotes, $\mathbf{c}$ immediately following intravenous injection of tdTomato $L$. donovani metacyclic promastigotes, d-f 6 days post-injection of tdTomato $L$. donovani amastigotes. g CFSElabeled memory-like OT-I T cells (green) 5-14 h post-transfer into d1421-infected hCD2.RFP mice (red T cells) infected with OVA-transgenic L. donovani. All images represent snapshots of the extended focus view of time-lapse imaging sequences. Associated videos are provided as Supplementary Movies 1-7. For further experimental details, see references [36, 37].
ROS production [40]. Although neutrophils were also observable patrolling the sinusoidal spaces in these imaging studies, they appeared to make little contact with $L$. donovani amastigotes and only rarely was phagocytosis directly observed. This is in contrast to the rapid engulfment of intravenously inoculated promastigotes of $L$. donovani by neutrophils (Fig. 1c and Supplementary Movie 3). The lack of immediate neutrophil swarming into the liver postinfection (a characteristic of promastigote inoculation; Beattie, unpublished) also confirmed the relative lack of inflammatory stimuli triggered by amastigotes under these conditions. Neutrophil indifference to amastigotes compared to promastigotes has also been reported for other Leishmania spp. in vitro [41].

Intravital imaging studies of BCG-infected mice showed a remarkably similar picture for the uptake of this mycobacterium; BCG was internalized by KCs within a minute or so of intravenous injection and became associated with intracellular compartments within 5 min of contact. Neutrophils, when observed patrolling the sinusoidal space, did not appear to engulf or contact BCG organisms in any appreciable manner, and this apparent indifference was also apparent even after the neutrophil influx characteristic of the early stages of BCGinduced inflammation [42].

\section{Chemokines and cytokines regulating early mononuclear cell recruitment}

Mononuclear cell recruitment into the nascent granuloma can occur by local repositioning of resident Kupffer cells or through the initiation of inflammation. Early during the process of granuloma formation, Kupffer cells may fuse, resulting in multinucleation [23] (Fig. 1d and Supplementary Movie 4). This process involves both infected and uninfected Kupffer cells, implying cues for local detachment and migration along the sinusoidal endothelium. Such Kupffer cell motility was also observed using BCG infection, and in both model systems, areas of Kupffer cell-rich granulomatous inflammation become interspersed with areas of the sinusoidal network devoid in their resident macrophages [37, 42]. Notably, the fate of Kupffer cells and their propensity to either form multinucleate cells or aggregates appears independent of parasite load, with some heavily infected Kupffer cells remaining as singletons in the highly inflamed liver environment for protracted periods of time (Fig. 1e and $\mathrm{f}$ and Supplementary Movies 5 and 6). This evident heterogeneity underlying granuloma formation provides a context for the development of computational simulations that aim to provide a mechanistic understanding of this observation (see below). Comparison with the behavior of Kupffer cells in BCG and $L$. donovani granulomas also serves to emphasize the relatively static nature of these cells/aggregates once within the core of the granuloma 
$[37,42]$, and although it is not possible to formally rule out, under the imaging conditions used, that some singletoninfected Kupffer cells arise from migration of Kupffer cells out of the granuloma environment, as noted in the granulomas formed in zebrafish infected with Mycobacterium marinarum [43], there is no current evidence to support this from studies in the immunologically intact mouse.

As infection proceeds past the first few hours, inflammatory cells begin to be recruited to the infected liver, and more specifically to infected Kupffer cells. Conventional approaches to examining the factors responsible for regulating tissue recruitment of inflammatory cells involves measurement of total tissue-derived chemokines/cytokines. We and others have used this approach to show early chemokine production, notably CCL2, CCL3, and CXCL10 [44-48]. In some instances, the functional importance of these factors has been validated by the use of gene-targeted mice in which there has been global loss of production of the factor under study. In common with similar studies using BCG and MTB, these experimental approaches provide valuable information about the cellular environment underlying the inflammatory event but fail to provide information regarding the cellspecific nature of these responses. Chemokines in particular are difficult to study by conventional immunohistochemical techniques, limiting the spatial information available. In some cases, ex vivo analysis of chemokine production has been performed following flow cytometry, but this has been on a rather piece-meal gene by gene basis. The importance of spatial information is exemplified by our analysis of CXCL10 regulation. Messenger RNA (mRNA) accumulation for $\mathrm{Ccl} 2, \mathrm{Ccl} 3$, and $\mathrm{Cxcl} 10$ was coordinately increased at $2 \mathrm{~h}$ postinfection in both wild-type mice and immunodeficient SCID or $\mathrm{Ragl}^{-/-}$mice [44, 48], and $C c l 2$ and $C c l 3$ mRNA returned to baseline levels by $24 \mathrm{~h}$ in both strains of mice [44]. In contrast, wild-type but not immunodeficient mice exhibited sustained accumulation of $C x c 110$ mRNA, indicative of the IFN $\gamma$-regulated nature of this chemokine. Surprisingly, however, given that IFN $\gamma$-producing NK cells, non-invariant NKT, and invariant NKT cells could all be detected in the liver at this time, studies in $\mathrm{J} \alpha 281^{-/}$mice indicated that only invariant NKT cells produced IFN $\gamma$ that was functionally relevant in this context [48].

In order to determine the global Kupffer cell response to infection, we developed an ex vivo transcriptomic profiling approach, wherein infection with TdTomato-expressing transgenic parasites allowed us to rapidly sort both infected and uninfected Kupffer cells from the liver of the same infected mouse. By comparison to Kupffer cells isolated from infected mice, this provided a unique opportunity to not only identify transcripts associated with the presence of intracellular amastigotes, but also to reveal how Kupffer cell activation proceeds in trans in response to inflammation [36]. This technique identified transcriptional signatures common to infected and "inflamed" Kupffer cells (largely mapping to GO terms associated with host defense and inflammatory response and validating many of the chemokine pathways identified earlier) and also signatures unique for infected cells (often associated with the regulation of macrophage gene expression and protein production). Surprisingly, we identified 241 transcripts uniquely regulated in the "inflamed" but not infected population of Kupffer cells. One explanation for this finding was that parasitism was favored by maintaining these genes at homeostatic levels even in the face of inflammation; hence, these genes might regulate a presumptive host defense pathway the parasite is avoiding. To test this hypothesis, we used pharmacologic intervention to perturb one of these regulatory gene networks, involved in lipid metabolism, and demonstrated that indeed this led to more effective Kupffer cell control of amastigote replication [36].

Other studies have examined the impact of gene $\mathrm{KO}$ on early myeloid cell recruitment. $C c r 5, C c l 3$, and $C c r 2 \mathrm{KO}$ mice all have $\mathrm{KC}$ aggregates of reduced size at 4 days p.i., with impaired granuloma organization measurable at 4 weeks [49]; similar defects were noted in a model of PPD-coated latex beadinduced lung granulomas in $\mathrm{Ccr} 2 \mathrm{KO}$ mice [50] and also in Ccrl KO mice in the Schistosoma egg granuloma model [51].

IL-33 is a Th2-associated cytokine that belongs to the alarmin family and that signals via the ST2 (Il1rl1)/IL-1 receptor accessory protein complex. IL-33 is produced by innate lymphoid cells and also by many non-hematopoietic cells. CCL2 and CXCL2 and their receptors (CCR2 and CXCR2) are increased in expression in hepatic tissues in infected Il1rll KO BALB/c mice coincident with enhanced Th1 responses and granuloma development and monocyte and neutrophil recruitment. These data suggest that signaling through ST2/ Illrl1 is a negative regulator of granuloma formation [52].

Cxcr3 KO mice, defective in responsiveness to CXCL9 and CXCL10, have delayed granulomatous responses but show otherwise effective antimicrobial function [53]; A similarly delayed response to aerosolized MTB infection was noted in Cxcr3 KO mice, suggesting that neutrophils were important for regulating early inflammation, though ultimately clearance of infection was obtained in these mice [54]; hence, $C x c r 3$ was not essential for immunity to $L$. donovani but allowed optimal recruitment of leucocytes to the granuloma [53].

$\mathrm{J} \propto 281 \mathrm{KO}$ mice defective in invariant NKT cells have delayed granuloma formation, and $90 \%$ of all chemokine, cytokine, and associated receptor genes upregulated in the livers of wild-type (WT) $L$. donovani-infected mice were found to be underexpressed in similarly infected $\mathrm{J} \alpha 18 \mathrm{KO}$ mice, underscoring the global deficiency in early stages of granuloma formation in these mice [55].

The precise upstream signaling that drives these inflammatory chemokine responses in infected and inflamed Kupffer cells is poorly characterized. Tlr2 $\mathrm{KO}$ mice infected with Leishmania amazonensis have eosinophilic granulomas in 
the early stages of infection compared to the mononuclear-rich responses of WT mice, suggesting that this TLR can modulate the balance of myeloid cell recruitment [56]. In $L$. donovani infection, TLR2 appears to negatively regulate granuloma formation, with more rapid granuloma maturation occurring in Tlr 2 KO mice; in contrast, TLR4 signaling appears to dampen the development of this response, and heavily parasitized granulomas persist. Importantly, the mechanisms of granuloma involution/resolution were not dysregulated in the absence of Tlr2, and such mice did not develop fulminating granulomatous pathology [57].

TLR9 is a PRR involved in the early recognition of Leishmania under some circumstances [58]. TLR9 expression has been demonstrated in granulomas from $L$. braziliensis patients but the evolution of this response was not studied and may relate to TLR9 regulation on macrophages after granuloma formation [59]. TLR9 has also been implicated in the initial response to L. infantum promastigotes in mice, acting as part of a regulatory network that controls NK cell IFN $\gamma$ production. The impact of Tlr 9 deficiency on granuloma formation was not, however, reported [58].

IRF5 can be activated downstream of TLR9/Myd88 and TLR7/Myd88 signaling. Irf5 KO mice infected with L. donovani have delayed $\mathrm{Th} 1$ responses, higher liver parasite burdens, and smaller Th2-biased granulomas with greater evidence of neutrophil involvement and concomitant accumulation of $\mathrm{Cxcll}$ mRNA in the granuloma [60]. A similar phenotype was also noted in $T l r 7 \mathrm{KO}$ mice. Although TLR7 operates via IRF7 as well as IRF5, no effect on splenic or $\mathrm{Th} 1$ responses (measured as $\mathrm{CD}^{+} \mathrm{T}$ cell-derived IFN $\gamma$ in the liver or spleen) was noted as a consequence of $\operatorname{Irf} 7$ deficiency in this study. In contrast, another study using Irf7 KO mice demonstrated a reduction in granuloma formation, Th1 response, and local NO response, along with an increase in the frequency of $\mathrm{CD}^{+} \mathrm{T}$ cells committed to producing IL-10 [61]. Exemplifying the divergent mechanisms of tissue and cellular control of Leishmania, Irf7-deficient Kupffer cells were shown to have an intact innate response to amastigotes of $L$. donovani, compared to the defective response seen in splenic marginal zone macrophages [61].

Given its role in regulating the Th1 response, the finding that Tbet-deficient mice are highly susceptible to $L$. donovani infection was not unexpected. Although granuloma formation proceeds in these mice, albeit in a restricted manner, most measures of Th1 immunity are significantly compromised. In contrast, and unexpectedly, Stat1-deficient mice are highly resistant to $L$. donovani infection, with minimal or absent granuloma formation in these mice [62]. Of note, granuloma formation was equivalent in Rag2 $\mathrm{KO}$ mice that received wild type or Stat1-deficient T cells by adoptive transfer, demonstrating that induction of granulomatous pathology was regulated through STAT1-dependent pathway(s) operating in nonlymphoid cells [62].
Although limited in the granulomas of wild type L. donovani-infected mice, there is increasing evidence that neutrophils regulate granuloma formation in experimental VL. Delayed granuloma formation was observed in mice depleted of neutrophils using mAb NIMP-R14, but this was far less of an effect than that observed on parasite load in the spleen and BM [63]. In B cell-deficient $\mu \mathrm{MT}$ mice, neutrophilic infiltration was exaggerated, leading to widespread hepatic necrosis, rarely seen in wild-type mice infected with L. donovani. In the context of B cell deficiency, neutrophilmediated pathology could be ameliorated by passive transfer of $\operatorname{Ig}[64]$.

\section{The maturing granuloma: a focus for cell-mediated immunity}

Analysis of the cytokine response in the maturing hepatic granuloma has been the focus of intense study for over three decades [65], and extensively reviewed on multiple occasions, often contrasting the host protective granulomatous response with the non-protective and non-granulomatous response seen in the spleen $[12,23,24,66,67]$. The reader is referred to these articles for a current overview. In summary, granulomatous inflammation appears the preferred route for focusing cytokine secretion onto infected macrophages, with the aim of stimulating the production of toxic anti-leishmanial species including nitric oxide and superoxide. Although Th1-like responses dominate (with IFN $\gamma$ being produced by multiple cell types) and alternatively activated macrophages are sparse in the L. donovani granuloma, the Th2-associated cytokines IL-4 and IL-13 are nevertheless required for optimal granuloma formation [68,69]. As Kupffer cells contain the vast bulk of amastigotes in the murine hepatic granuloma [37], it is likely that this macrophage population is responsible for parasite elimination. However, it remains possible that Kupffer cell lysis occurs and that leishmanicidal activity is delivered by granuloma-infiltrating monocytes or neutrophils that rapidly phagocytose the released amastigotes.

It is worth bearing in mind, however, some of the experimental limitations of many of these studies, when it comes to precisely assigning the role of cytokines and/or cells within the microenvironment of the granuloma per se. For example, technical challenges associated with the small size and limited structural integrity of the L. donovani granuloma makes isolation difficult and often whole liver cell populations are used for analysis as a surrogate. Alternatively, the experimental intervention may affect immune regulation within the granuloma, but it also likely has more global effects (e.g. consider the mouse treated with $\mathrm{mAb}$ to neutralize a given cytokine), that indirectly affect granuloma form or function. Finally, while granuloma heterogeneity (cellularity, location, or apparent microbicidal activity) is clearly evident even by simple 
histological analysis, aggregation of data from this heterogeneous population is the current norm. Unlike studies on TB, where ex vivo access to and/or imaging of individual granulomas in situ is possible [70-72], our ability to interrogate the individual granuloma in leishmaniasis patients and animal models remains limited at present.

Attempts to isolate the hepatic environment from other systemic sites responding to their own "local" Leishmania infection have provided some insights into the independence of hepatic immunity, but largely not addressed the challenge of defining responses within the granuloma microenvironment per se. For example, in aly/aly mice that lack LN, Peyer's patches, and have disrupted splenic architecture, granulomas are generated in the liver [73]. This is in keeping with recent data showing that splenectomized LT-deficient mice (also lacking LNs, hence devoid of conventional secondary lymphoid tissue) have the capacity to mount immune responses against this infection including normal granuloma formation [74]. In the case of aly/aly mice, parasites persist within granulomas accompanied by an influx of Foxp $3^{+}$Tregs that is not seen in granulomas of WT mice [73].

Intravital microscopy provides the opportunity to study individual granulomas in situ in live animals, but imposes its own constraints. Imaging windows are short, usually a few hours at most, and the procedures used are terminal, limiting the observation of granulomas over realistic time periods to observe their maturation. Observation is generally limited to one or a small number of cell populations, and the functional consequences of those interactions, be that intracellular signaling events or cytokine production, are only recently becoming amenable to imaging [75]. Nevertheless, the study of $\mathrm{CD}^{+} \mathrm{T}$ cell $[42,76,77]$ and $\mathrm{CD} 8^{+} \mathrm{T}$ cell behavior within the BCG and L. donovani $[37,78]$ granuloma has provided valuable novel insights into local effector $\mathrm{T}$ cell activity (Fig. $1 \mathrm{~g}$ and Supplementary Movie 7). In spite of these studies focusing on different $T$ cell subsets, commonalities emerge: (i) both antigen-specific and non-specific $\mathrm{T}$ cells are recruited into the granuloma - a not unsurprising finding given the inflammatory nature of this environment, but one that serves as a reminder that enumeration of all $\mathrm{T}$ cells in the granuloma is not a likely measure of effector function; (ii) $\mathrm{T}$ cells move freely into and out of granulomas, with no evident wallingoff of the granuloma from its external environment; (iii) within granulomas $\mathrm{T}$ cell velocity was similar to that seen in organized lymphoid tissues, but other measures of $\mathrm{T}$ cell migration indicated some local constraints on free migration within the confines of a granuloma; (iv) long-term contacts between $\mathrm{T}$ cells and APC, indicative of cognate interactions and measurable by a dwell time for granuloma exit, was observed for a minority of antigen-specific T cells, suggesting local presentation of cognate antigen was limiting. This conclusion is supported in the case of $\mathrm{CD} 4^{+} \mathrm{T}$ cells by demonstrating arrest of a much greater frequency of intra-granuloma $\mathrm{T}$ cells after exogenous administration of peptide [76]. Within the L. donovani granuloma, expression of MHCI-peptide complexes was an almost exclusive property of infected Kupffer cells and such cells formed the focus of attention for $\mathrm{CD}^{+}$T cells [37]; and (v) constraints on APC efficiency within the granuloma may reflect limited MHCpeptide complex expression coupled to local pathogen load [76, 77] or altered expression of checkpoint inhibitors [79].

These studies provide a basis for future examination of other aspects of granuloma biology that may be pertinent to understanding disease chronicity and or targeted therapeutic intervention. For example, whether $\mathrm{T}$ cell behavior remains similar across the spectrum of granuloma heterogeneity is unknown, and how changing features of the non-lymphoid compartment of the granuloma including progressive fibrosis, may affect T cell-APC interactions would appear to be an essential route of future investigation. Likewise, the role of checkpoint inhibitors as host-directed therapy has a proven experimental base (see below) but whether this occurs through direct manipulation of $\mathrm{T}$ cell biology in the granuloma remains to be established. As pointed out by Germain and colleagues [76], manipulation of local APC function may prove more profitable than or be an effective adjunct to enhancing effector T cell frequency, and in situ analysis of the type described above may provide the most useful means to monitor the success of such interventions.

\section{Computational simulation of granuloma development and function}

To overcome some of these experimental limitations and to gain further insight into granuloma heterogeneity, dynamics, and function, we and others have also adopted computational approaches. In TB, these models have focused largely on understanding macrophage polarization and the role of TNF [80-82].

Our approach in modeling the $L$. donovani granuloma has been twofold - first, the generation of agent-based models of early granuloma formation and, second, the use of Petri net modeling of granuloma heterogeneity and within granuloma immune interactions. The former have been discussed elsewhere [83] and will not be considered further here. The Petri net model that we have used to evaluate the development of granulomas and the emergent immune regulation that influences parasite load is not spatially explicit and assumes no structural organization within the granuloma per se. It has, however, provided invaluable insights into potential sources of granuloma heterogeneity and allowed us to postulate the presence of a small subset of granulomas that have altered immune regulatory function that leads to parasite persistence [84]. Such granulomas may well be a source of recrudescence upon immune suppression, a common feature of experimental models of VL and also seen in human disease, and the 
generation of new hypotheses amenable to experimental validation is arguably one of the central roles that modeling can play in understanding complex, dynamic, and long-lived disease processes.

\section{The granuloma as a tertiary lymphoid organ}

Tertiary lymphoid organs are commonplace in conditions of chronic inflammation, representing accumulations of lymphoid cells and stromal cells that recapitulate aspects of lymphoid tissue architecture [85]. In autoimmune diseases, the production of tertiary lymphoid organs (TLOs) is increasingly recognized as a poor prognostic indicator, serving to perpetuate the immune response. Lung TLO's have also been reported in mice infected via aerosol with Mycobacterium tuberculosis $\mathrm{H} 37 \mathrm{RV}$ and in human tuberculosis, associated with granulomas [86-88]. Central to the formation of TLOs is the lymphotoxin-dependent appearance of stromal cells producing constitutive chemokines associated with lymphocyte aggregation. Notably, most TLOs express CXCL13, the chemokine produced by follicular dendritic cells and responsible for B cell aggregation in germinal centers [89]. Hence, curtailment of the production and maintenance of TLOs is viewed as a potential target for stromal cell-targeted host-directed therapy. Although B cells are found within the granulomas of $L$. donovani-infected mice, and these make sustained interactions with granuloma $\mathrm{T}$ cells that are suggestive of cognate interactions [90], we have not found evidence for TLO-like organization either within the granuloma itself or within the hepatic microenvironment during $L$. donovani infection. CXCL13 expression was not detectable nor were cells expressing the FDC marker FDCM1. The reasons for the lack of TLO formation during $L$. donovani infection are currently unknown. One possibility is that the relative paucity of strong PAMPs in Leishmania compared to MTB limits the extent of underlying innate immune responses that drive TLO formation. On the other hand, the relative ease with which Leishmania succumb to macrophage leishmanicidal activity may allow premature truncation of the infection before TLOs can fully develop. Alternatively, Leishmania infections may not provide appropriate signals for the recruitment and activation of lymphoid tissue inducer (LTi) cells. LTi cells are a necessary precursor to TLO formation. In support of this latter possibility, whereas restoration of lymphoid tissue architecture following MCMV infection is dependent upon LTi function [91], such cells do not appear to play a role in restoration of lymphoid tissue architecture in $L$. donovani-infected mice treated with sunitinib maleate, a receptor tyrosine kinase inhibitor that restores stromal cell structure and function [92].

\section{Host-directed therapies targeting granulomatous inflammation}

The therapeutic benefit of modulating key pathways in immune regulation on the outcome of $L$. donovani infection provided some of the earliest examples of the use of checkpoint inhibitors, now an increasingly popular clinical intervention strategy for cancer and autoimmunity [93, 94], as tools to manipulate immunity to pathogen infection. Following observations that the expression of costimulatory molecules of the B7 family were reduced in expression on infected Kupffer cells at the core of the granuloma [79] and on L. donovaniinfected macrophages in vitro [95], mAb targeting costimulatory pathways were examined for their ability to affect granuloma formation and host protective immunity. Administration of a blocking antibody to B7-2 (CD86) had no impact on early immune or inflammatory responses, whereas later administration led to enhanced Th1 responses and granuloma formation. These data suggested that late CD86-CTLA4 interactions might limit the expression of maximum immunity to L. donovani [96]. Confirming this prediction, administration of a mAb (4F10) targeting CTLA-4 was shown to significantly accelerate granuloma formation and lead to significant reductions in tissue parasite burden. Notably, anti-CTLA4 could be used therapeutically in mice with established infection, either alone [97] or in a synergistic fashion with conventional chemotherapy $[98,99]$. Other checkpoint regulators including OX40L-Fc [98], anti-CD40 [99], and PDL-1 [100] have proven equally effective at enhancing granuloma formation and protective immunity in chronically infected mice, in the absence of any overtly tissue-damaging pathology. To date, no clinical trials of such checkpoint inhibitors have been performed in the context of leishmaniasis, although evidence for defects associated with dysregulated checkpoint control, e.g., $\mathrm{CD} 8^{+} \mathrm{T}$ cell exhaustion, have been obtained in the clinic [101]. Attempts to validate the use of checkpoint-regulating antibodies with in vitro cultures of infected macrophages and $\mathrm{T}$ cells have had mixed results, with the PD-1 pathway blockade inducing heightened macrophage activation and parasite killing in cells obtained from active cases of canine VL [102] but not human VL [101].

The success of checkpoint blockade in models of L. donovani infection is not mirrored in models of mycobacterial infection. For example, whereas CTLA4 blockade significantly increased multiple correlates of immunity in mice infected with BCG, there was no impact on mycobacterial load in the spleen, liver, or lung and no changes to lung histopathology and granuloma formation [103]. Importantly, removal of regulatory control over $\mathrm{T}$ cell activation during mycobacterial infection may lead to the development of overt pathology, as in the case of manipulation of the PD-1 pathway [104]. It is likely that differences in outcome of checkpoint regulation related to pathology vs. protection reflect the 
relative ease with which Leishmania succumbs to the antimicrobial defenses compared to mycobacteria and to differences in the inherent immunogenicity of these organisms, with Leishmania being a renowned "silent invader" [3]. These characteristics may suggest a brighter future for checkpoint inhibitors as therapeutic agents in human leishmaniasis compared to mycobacterial disease.

Acknowledgments Research in the authors' laboratories is funded by the UK Medical Research Council (to PMK) and The Australian Infectious Diseases research network and QIMRB (to LB). The authors thank all those in the Kaye laboratory, past and present, that have contributed to the understanding of granulomatous pathology in experimental leishmaniasis. The authors also apologize to those whose work was not cited due to constraints on space.

\section{Compliance with ethical standards}

Conflict of interest The authors declare that they have no competing interests.

Open Access This article is distributed under the terms of the Creative Commons Attribution 4.0 International License (http:// creativecommons.org/licenses/by/4.0/), which permits unrestricted use, distribution, and reproduction in any medium, provided you give appropriate credit to the original author(s) and the source, provide a link to the Creative Commons license, and indicate if changes were made.

\section{References}

1. Ridley MJ, Ridley DS (1986) Monocyte recruitment, antigen degradation and localization in cutaneous leishmaniasis. Br J Exp Pathol 67:209-218

2. Alvar J, Velez ID, Bern C, Herrero M, Desjeux P et al (2012) Leishmaniasis worldwide and global estimates of its incidence. PLoS One 7, e35671

3. Kaye P, Scott P (2011) Leishmaniasis: complexity at the hostpathogen interface. Nat Rev Microbiol 9:604-615

4. Lukes J, Mauricio IL, Schonian G, Dujardin JC, Soteriadou K et al (2007) Evolutionary and geographical history of the Leishmania donovani complex with a revision of current taxonomy. Proc Natl Acad Sci U S A 104:9375-9380

5. Tuon FF, Neto VA, Amato VS (2008) Leishmania: origin, evolution and future since the Precambrian. FEMS Immunol Med Microbiol 54:158-166

6. Aoun J, Habib R, Charaffeddine K, Taraif S, Loya A et al (2014) Caseating granulomas in cutaneous leishmaniasis. PLoS Negl Trop Dis 8, e3255

7. Elso C, Kumar B, Smyth G, Foote S, Handman E (2004) Dissociation of disease susceptibility, inflammation and cytokine profile in $1 \mathrm{mr} 1 / 2$ congenic mice infected with Leishmania major. Genes Immun 5:188-196

8. Elso CM, Roberts LJ, Smyth GK, Thomson RJ, Baldwin TM et al (2004) Leishmaniasis host response loci (lmr1-3) modify disease severity through a Th1/Th2-independent pathway. Genes Immun 5:93-100

9. Gutierrez Y, Salinas GH, Palma G, Valderrama LB, Santrich CV et al (1991) Correlation between histopathology, immune response, clinical presentation, and evolution in Leishmania braziliensis infection. Am J Trop Med Hyg 45:281-289
10. Souza-Lemos C, de-Campos SN, Teva A, Corte-Real S, Fonseca $\mathrm{EC}$ et al (2008) Dynamics of immune granuloma formation in a Leishmania braziliensis-induced self-limiting cutaneous infection in the primate Macaca mulatta. J Pathol 216:375-386

11. Souza-Lemos C, de-Campos SN, Teva A, Porrozzi R, Grimaldi G $\mathrm{Jr}$ (2011) In situ characterization of the granulomatous immune response with time in nonhealing lesional skin of Leishmania braziliensis-infected rhesus macaques (Macaca mulatta). Vet Immunol Immunopathol 142:147-155

12. Kaye PM, Svensson M, Ato M, Maroof A, Polley R et al (2004) The immunopathology of experimental visceral leishmaniasis. Immunol Rev 201:239-253

13. Moreno A, Marazuela M, Yebra M, Hernandez MJ, Hellin T et al (1988) Hepatic fibrin-ring granulomas in visceral leishmaniasis. Gastroenterology 95:1123-1126

14. Geramizadeh B, Jahangiri R, Moradi E (2011) Causes of hepatic granuloma: a 12-year single center experience from southern Iran. Arch Iran Med 14:288-289

15. Zijlstra EE, el-Hassan AM (2001) Leishmaniasis in Sudan. Visceral leishmaniasis. Trans R Soc Trop Med Hyg 95(Suppl 1): S27-S58

16. Chandra H, Chandra S, Kaushik RM (2013) Visceral leishmaniasis with associated common, uncommon, and atypical morphological features on bone marrow aspirate cytology in nonendemic region. J Trop Med 2013:861032

17. Dhingra KK, Gupta P, Saroha V, Setia N, Khurana N et al (2010) Morphological findings in bone marrow biopsy and aspirate smears of visceral Kala Azar: a review. Indian J Pathol Microbiol 53:96-100

18. Pampiglione S, Manson-Bahr PE, Giungi F, Giunti G, Parenti A et al (1974) Studies on Mediterranean leishmaniasis 2. Asymptomatic cases of visceral leishmaniasis. Trans R Soc Trop Med Hyg 68:447-453

19. dos-Santos WL, David J, Badaro R, de-Freitas LA (2004) Association between skin parasitism and a granulomatous inflammatory pattern in canine visceral leishmaniosis. Parasitol Res 92: 89-94

20. Sanchez MA, Diaz NL, Zerpa O, Negron E, Convit J et al (2004) Organ-specific immunity in canine visceral leishmaniasis: analysis of symptomatic and asymptomatic dogs naturally infected with Leishmania chagasi. Am J Trop Med Hyg 70: 618-624

21. Laurenti MD, Sotto MN, Corbett CE, da Matta VL, Duarte MI (1990) Experimental visceral leishmaniasis: sequential events of granuloma formation at subcutaneous inoculation site. Int J Exp Pathol 71:791-797

22. Wilson ME, Innes DJ, Sousa AD, Pearson RD (1987) Early histopathology of experimental infection with Leishmania donovani in hamsters. J Parasitol 73:55-63

23. Murray HW (2001) Tissue granuloma structure-function in experimental visceral leishmaniasis. Int J Exp Pathol 82:249-267

24. Engwerda CR, Kaye PM (2000) Organ-specific immune responses associated with infectious disease. Immunol Today 21: 73-78

25. Ghosh MK, Nandy A, Addy M, Maitra TK, Ghose AC (1995) Subpopulations of T lymphocytes in the peripheral blood, dermal lesions and lymph nodes of post kala-azar dermal leishmaniasis patients. Scand J Immunol 41:11-17

26. Khalil EA, Khidir SA, Musa AM, Musa BY, Elfaki ME et al (2013) Post-kala-azar dermal leishmaniasis: a paradigm of paradoxical immune reconstitution syndrome in non-HIV/AIDS patients. J Trop Med 2013:275253

27. Desjeux P, Ghosh RS, Dhalaria P, Strub-Wourgaft N, Zijlstra EE (2013) Report of the post kala-azar dermal leishmaniasis (PKDL) consortium meeting, New Delhi, India, 27-29 June 2012. Parasit Vectors 6:196 
28. Mukhopadhyay D, Dalton JE, Kaye PM, Chatterjee M (2014) Post kala-azar dermal leishmaniasis: an unresolved mystery. Trends Parasitol 30:65-74

29. Zijlstra EE, el-Hassan AM (2001) Leishmaniasis in Sudan. Post kala-azar dermal leishmaniasis. Trans R Soc Trop Med Hyg 95(Suppl 1):S59-S76

30. Zijlstra EE, Musa AM, Khalil EA, el-Hassan IM, el-Hassan AM (2003) Post-kala-azar dermal leishmaniasis. Lancet Infect Dis 3:87-98

31. Mukherjee S, Mukhopadhyay D, Braun C, Barbhuiya JN, Das NK et al (2015) Decreased presence of Langerhans cells is a critical determinant for Indian post kala-azar dermal leishmaniasis. Exp Dermatol 24:232-234

32. Menard R, Tavares J, Cockburn I, Markus M, Zavala F et al (2013) Looking under the skin: the first steps in malarial infection and immunity. Nat Rev Microbiol 11:701-712

33. McElrath MJ, Murray HW, Cohn ZA (1988) The dynamics of granuloma formation in experimental visceral leishmaniasis. J Exp Med 167:1927-1937

34. Gomez Perdiguero E, Geissmann F (2013) Myb-independent macrophages: a family of cells that develops with their tissue of residence and is involved in its homeostasis. Cold Spring Harb Symp Quant Biol 78:91-100

35. Schulz C, Gomez Perdiguero E, Chorro L, Szabo-Rogers H, Cagnard N et al (2012) A lineage of myeloid cells independent of Myb and hematopoietic stem cells. Science 336:86-90

36. Beattie L, d'El-Rei Hermida M, Moore JW, Maroof A, Brown N et al (2013) A transcriptomic network identified in uninfected macrophages responding to inflammation controls intracellular pathogen survival. Cell Host Microbe 14:357-368

37. Beattie L, Peltan A, Maroof A, Kirby A, Brown N et al (2010) Dynamic imaging of experimental Leishmania donovani-induced hepatic granulomas detects Kupffer cell-restricted antigen presentation to antigen-specific CD8 T cells. PLoS Pathog 6, e1000805

38. Ueno N, Wilson ME (2012) Receptor-mediated phagocytosis of Leishmania: implications for intracellular survival. Trends Parasitol 28:335-344

39. Hsiao CH, Ueno N, Shao JQ, Schroeder KR, Moore KC et al (2011) The effects of macrophage source on the mechanism of phagocytosis and intracellular survival of Leishmania. Microbes Infect 13:1033-1044

40. Lefevre L, Lugo-Villarino G, Meunier E, Valentin A, Olagnier D et al (2013) The C-type lectin receptors dectin-1, MR, and SIGNR3 contribute both positively and negatively to the macrophage response to Leishmania infantum. Immunity 38:1038-1049

41. Wenzel UA, Bank E, Florian C, Forster S, Zimara N et al (2012) Leishmania major parasite stage-dependent host cell invasion and immune evasion. FASEB J 26:29-39

42. Egen JG, Rothfuchs AG, Feng CG, Winter N, Sher A et al (2008) Macrophage and $\mathrm{T}$ cell dynamics during the development and disintegration of mycobacterial granulomas. Immunity 28:271-284

43. Davis JM, Ramakrishnan L (2009) The role of the granuloma in expansion and dissemination of early tuberculous infection. Cell 136:37-49

44. Cotterell SE, Engwerda CR, Kaye PM (1999) Leishmania donovani infection initiates $\mathrm{T}$ cell-independent chemokine responses, which are subsequently amplified in a T cell-dependent manner. Eur J Immunol 29:203-214

45. Gupta $G$, Bhattacharjee S, Bhattacharyya S, Bhattacharya $P$, Adhikari A et al (2009) CXC chemokine-mediated protection against visceral leishmaniasis: involvement of the proinflammatory response. J Infect Dis 200:1300-1310

46. Gupta G, Majumdar S, Adhikari A, Bhattacharya P, Mukherjee AK et al (2011) Treatment with IP-10 induces host-protective immune response by regulating the $\mathrm{T}$ regulatory cell functioning in Leishmania donovani-infected mice. Med Microbiol Immunol 200:241-253

47. Majumder S, Bhattacharjee S, Paul Chowdhury B, Majumdar S (2012) CXCL10 is critical for the generation of protective CD8 T cell response induced by antigen pulsed $\mathrm{CpG}-\mathrm{ODN}$ activated dendritic cells. PLoS One 7, e48727

48. Svensson M, Zubairi S, Maroof A, Kazi F, Taniguchi M et al (2005) Invariant NKT cells are essential for the regulation of hepatic CXCL10 gene expression during Leishmania donovani infection. Infect Immun 73:7541-7547

49. Sato N, Kuziel WA, Melby PC, Reddick RL, Kostecki V et al (1999) Defects in the generation of IFN-gamma are overcome to control infection with Leishmania donovani in CC chemokine receptor (CCR) 5-, macrophage inflammatory protein-1 alpha-, or CCR2-deficient mice. J Immunol 163:5519-5525

50. Boring L, Gosling J, Chensue SW, Kunkel SL, Farese RV Jr et al (1997) Impaired monocyte migration and reduced type 1 (Th1) cytokine responses in C-C chemokine receptor 2 knockout mice. J Clin Invest 100:2552-2561

51. Gao JL, Wynn TA, Chang Y, Lee EJ, Broxmeyer HE et al (1997) Impaired host defense, hematopoiesis, granulomatous inflammation and type 1-type 2 cytokine balance in mice lacking CC chemokine receptor 1. J Exp Med 185:1959-1968

52. Rostan O, Gangneux JP, Piquet-Pellorce C, Manuel C, McKenzie AN et al (2013) The IL-33/ST2 axis is associated with human visceral leishmaniasis and suppresses Th1 responses in the livers of BALB/c mice infected with Leishmania donovani. MBio 4: e00383-00313

53. Barbi J, Oghumu S, Rosas LE, Carlson T, Lu B et al (2007) Lack of CXCR3 delays the development of hepatic inflammation but does not impair resistance to Leishmania donovani. J Infect Dis 195:1713-1717

54. Seiler P, Aichele P, Bandermann S, Hauser AE, Lu B et al (2003) Early granuloma formation after aerosol Mycobacterium tuberculosis infection is regulated by neutrophils via CXCR3-signaling chemokines. Eur J Immunol 33:2676-2686

55. Robert-Gangneux F, Drogoul AS, Rostan O, Piquet-Pellorce C, Cayon J et al (2012) Invariant NKT cells drive hepatic cytokinic microenvironment favoring efficient granuloma formation and early control of Leishmania donovani infection. PLoS One 7, e33413

56. Guerra CS, Silva RM, Carvalho LO, Calabrese KS, Bozza PT et al (2010) Histopathological analysis of initial cellular response in TLR-2 deficient mice experimentally infected by Leishmania (L.) amazonensis. Int J Exp Pathol 91:451-459

57. Murray HW, Zhang Y, Zhang Y, Raman VS, Reed SG et al (2013) Regulatory actions of Toll-like receptor 2 (TLR2) and TLR4 in Leishmania donovani infection in the liver. Infect Immun 81: 2318-2326

58. Schleicher U, Liese J, Knippertz I, Kurzmann C, Hesse A et al (2007) NK cell activation in visceral leishmaniasis requires TLR9, myeloid DCs, and IL-12, but is independent of plasmacytoid DCs. J Exp Med 204:893-906

59. Tuon FF, Fernandes ER, Pagliari C, Duarte MI, Amato VS (2010) The expression of TLR9 in human cutaneous leishmaniasis is associated with granuloma. Parasite Immunol 32:769-772

60. Paun A, Bankoti R, Joshi T, Pitha PM, Stager S (2011) Critical role of IRF-5 in the development of T helper 1 responses to Leishmania donovani infection. PLoS Pathog 7, e1001246

61. Beattie L, Phillips R, Brown N, Owens BM, Chauhan N et al (2011) Interferon regulatory factor 7 contributes to the control of Leishmania donovani in the mouse liver. Infect Immun 79: 1057-1066

62. Rosas LE, Snider HM, Barbi J, Satoskar AA, Lugo-Villarino G et al (2006) Cutting edge: STAT1 and T-bet play distinct roles in 
determining outcome of visceral leishmaniasis caused by Leishmania donovani. J Immunol 177:22-25

63. McFarlane E, Perez C, Charmoy M, Allenbach C, Carter KC et al (2008) Neutrophils contribute to development of a protective immune response during onset of infection with Leishmania donovani. Infect Immun 76:532-541

64. Smelt SC, Cotterell SE, Engwerda CR, Kaye PM (2000) B celldeficient mice are highly resistant to Leishmania donovani infection, but develop neutrophil-mediated tissue pathology. J Immunol 164:3681-3688

65. Murray HW (1982) Cell-mediated immune response in experimental visceral leishmaniasis. II. Oxygen-dependent killing of intracellular Leishmania donovani amastigotes. J Immunol 129: 351-357

66. Engwerda CR, Ato M, Kaye PM (2004) Macrophages, pathology and parasite persistence in experimental visceral leishmaniasis. Trends Parasitol 20:524-530

67. Faleiro RJ, Kumar R, Hafner LM, Engwerda CR (2014) Immune regulation during chronic visceral leishmaniasis. PLoS Negl Trop Dis 8, e2914

68. McFarlane E, Carter KC, McKenzie AN, Kaye PM, Brombacher F et al (2011) Endogenous IL-13 plays a crucial role in liver granuloma maturation during Leishmania donovani infection, independent of IL-4R alpha-responsive macrophages and neutrophils. J Infect Dis 204:36-43

69. Stager S, Alexander J, Carter KC, Brombacher F, Kaye PM (2003) Both interleukin-4 (IL-4) and IL-4 receptor alpha signaling contribute to the development of hepatic granulomas with optimal antileishmanial activity. Infect Immun 71:4804 4807

70. Chen RY, Dodd LE, Lee M, Paripati P, Hammoud DA et al (2014) $\mathrm{PET} / \mathrm{CT}$ imaging correlates with treatment outcome in patients with multidrug-resistant tuberculosis. Sci Transl Med 6:265ra166

71. Coleman MT, Chen RY, Lee M, Lin PL, Dodd LE et al (2014) $\mathrm{PET} / \mathrm{CT}$ imaging reveals a therapeutic response to oxazolidinones in macaques and humans with tuberculosis. Sci Transl Med 6: 265ra167

72. Gideon HP, Phuah J, Myers AJ, Bryson BD, Rodgers MA et al (2015) Variability in tuberculosis granuloma T cell responses exists, but a balance of pro- and anti-inflammatory cytokines is associated with sterilization. PLoS Pathog 11, e1004603

73. Tiwananthagorn S, Iwabuchi K, Ato M, Sakurai T, Kato H et al (2012) Involvement of CD4(+) Foxp3(+) regulatory T cells in persistence of Leishmania donovani in the liver of alymphoplastic aly/aly mice. PLoS Negl Trop Dis 6, e1798

74. Bunn PT, Stanley AC, de Labastida RF, Mulherin A, Sheel M et al (2014) Tissue requirements for establishing long-term CD4+ T cell-mediated immunity following Leishmania donovani infection. J Immunol 192:3709-3718

75. Kong L, Tang J, Little JP, Yu Y, Lammermann T et al (2015) Continuous volumetric imaging via an optical phase-locked ultrasound lens. Nat Methods 12:759-762

76. Egen JG, Rothfuchs AG, Feng CG, Horwitz MA, Sher A et al (2011) Intravital imaging reveals limited antigen presentation and $\mathrm{T}$ cell effector function in mycobacterial granulomas. Immunity 34:807-819

77. Torabi-Parizi P, Vrisekoop N, Kastenmuller W, Gerner MY, Egen JG et al (2014) Pathogen-related differences in the abundance of presented antigen are reflected in CD4+ T cell dynamic behavior and effector function in the lung. J Immunol 192:1651-1660

78. Beattie L, Kaye PM (2011) Leishmania-host interactions: what has imaging taught us? Cell Microbiol 13:1659-1667

79. Kaye PM, Rogers NJ, Curry AJ, Scott JC (1994) Deficient expression of co-stimulatory molecules on Leishmania-infected macrophages. Eur J Immunol 24:2850-2854

80. Cilfone NA, Perry CR, Kirschner DE, Linderman JJ (2013) Multiscale modeling predicts a balance of tumor necrosis factor-alpha and interleukin-10 controls the granuloma environment during Mycobacterium tuberculosis infection. PLoS One 8, e68680

81. Day J, Friedman A, Schlesinger LS (2009) Modeling the immune rheostat of macrophages in the lung in response to infection. Proc Natl Acad Sci U S A 106:11246-11251

82. Marino S, Cilfone NA, Mattila JT, Linderman JJ, Flynn JL et al (2015) Macrophage polarization drives granuloma outcome during Mycobacterium tuberculosis infection. Infect Immun 83:324-338

83. Moore JW, Moyo D, Beattie L, Andrews PS, Timmis J et al (2013) Functional complexity of the Leishmania granuloma and the potential of in silico modeling. Front Immunol 4:35

84. Albergante L, Timmis J, Beattie L, Kaye PM (2013) A Petri net model of granulomatous inflammation: implications for IL-10 mediated control of Leishmania donovani infection. PLoS Comput Biol 9, e1003334

85. Carragher DM, Rangel-Moreno J, Randall TD (2008) Ectopic lymphoid tissues and local immunity. Semin Immunol 20:26-42

86. Kahnert A, Hopken UE, Stein M, Bandermann S, Lipp M et al (2007) Mycobacterium tuberculosis triggers formation of lymphoid structure in murine lungs. J Infect Dis 195:46-54

87. Maglione PJ, Xu J, Chan J (2007) B cells moderate inflammatory progression and enhance bacterial containment upon pulmonary challenge with Mycobacterium tuberculosis. J Immunol 178: $7222-7234$

88. Ulrichs T, Kosmiadi GA, Trusov V, Jorg S, Pradl L et al (2004) Human tuberculous granulomas induce peripheral lymphoid follicle-like structures to orchestrate local host defence in the lung. J Pathol 204:217-228

89. Buckley CD, Barone F, Nayar S, Benezech C, Caamano J (2015) Stromal cells in chronic inflammation and tertiary lymphoid organ formation. Annu Rev Immunol 33:715-745

90. Moore JW, Beattie L, Dalton JE, Owens BM, Maroof A et al (2012) B cell: T cell interactions occur within hepatic granulomas during experimental visceral leishmaniasis. PLoS One 7, e34143

91. Scandella E, Bolinger B, Lattmann E, Miller S, Favre S et al (2008) Restoration of lymphoid organ integrity through the interaction of lymphoid tissue-inducer cells with stroma of the T cell zone. Nat Immunol 9:667-675

92. Dalton JE, Maroof A, Owens BM, Narang P, Johnson K et al (2010) Inhibition of receptor tyrosine kinases restores immunocompetence and improves immune-dependent chemotherapy against experimental leishmaniasis in mice. J Clin Invest 120: 1204-1216

93. Sharma P, Allison JP (2015) The future of immune checkpoint therapy. Science 348:56-61

94. Topalian SL, Drake CG, Pardoll DM (2015) Immune checkpoint blockade: a common denominator approach to cancer therapy. Cancer Cell 27:450-461

95. Saha B, Das G, Vohra H, Ganguly NK, Mishra GC (1995) Macrophage-T cell interaction in experimental visceral leishmaniasis: failure to express costimulatory molecules on Leishmaniainfected macrophages and its implication in the suppression of cell-mediated immunity. Eur J Immunol 25:2492-2498

96. Murphy ML, Engwerda CR, Gorak PM, Kaye PM (1997) B7-2 blockade enhances $\mathrm{T}$ cell responses to Leishmania donovani. J Immunol 159:4460-4466

97. Murphy ML, Cotterell SE, Gorak PM, Engwerda CR, Kaye PM (1998) Blockade of CTLA-4 enhances host resistance to the intracellular pathogen, Leishmania donovani. J Immunol 161: 4153-4160

98. Zubairi S, Sanos SL, Hill S, Kaye PM (2004) Immunotherapy with OX40L-Fc or anti-CTLA-4 enhances local tissue responses and killing of Leishmania donovani. Eur J Immunol 34: $1433-1440$ 
99. Murray HW, Lu CM, Brooks EB, Fichtl RE, DeVecchio JL et al (2003) Modulation of T-cell costimulation as immunotherapy or immunochemotherapy in experimental visceral leishmaniasis. Infect Immun 71:6453-6462

100. Joshi T, Rodriguez S, Perovic V, Cockburn IA, Stager S (2009) B7-H1 blockade increases survival of dysfunctional CD8(+) T cells and confers protection against Leishmania donovani infections. PLoS Pathog 5, e1000431

101. Gautam S, Kumar R, Singh N, Singh AK, Rai M et al (2014) CD8 $\mathrm{T}$ cell exhaustion in human visceral leishmaniasis. J Infect Dis 209:290-299
102. Esch KJ, Juelsgaard R, Martinez PA, Jones DE, Petersen CA (2013) Programmed death 1-mediated T cell exhaustion during visceral leishmaniasis impairs phagocyte function. J Immunol 191:5542-5550

103. Kirman J, McCoy K, Hook S, Prout M, Delahunt B et al (1999) CTLA-4 blockade enhances the immune response induced by mycobacterial infection but does not lead to increased protection. Infect Immun 67:3786-3792

104. Barber DL, Mayer-Barber KD, Feng CG, Sharpe AH, Sher A (2011) CD4 T cells promote rather than control tuberculosis in the absence of PD-1-mediated inhibition. J Immunol 186: $1598-1607$ 